\title{
Über eine Nucleinsäure aus der Pankreasdrüse.
}

\author{
I. Mitteilung. \\ Von \\ R. Feulgen. \\ (Aus dem physiologischen Institut der Universität Berlin.) \\ (Der Redaktion zugegangen am 6. November 1913.)
}

Unsere Kenntnisse über die Zusammensetzung und den Bau der echten Nucleinsäuren sind hauptsächlich an Substanzen gewonnen worden, die aus den Kernen der Thymusdrüse vom Kalb oder aus den Köpfen von Fischspermien erhalten werden. Die Gründe hierfür liegen einmal darin, daß aus diesen Organen. die Nucleinsäure in guten Ausbeuten erhalten wird, und zweitens darin, daß keine anderen Substanzen, die der Gruppe der Nucleinsäuren zuzurechnen wären, bisher aus ihnen isoliert worden sind.

Bei anderen Organen, wie Leber, Milz, Pankreas usw. liegen die Verhältnisse komplizierter; hier sind die Ausbeuten an Körpern, die der echten Nucleinsäure gleichen, verschwindend klein, und überdies besteht immer die Möglichkeit, daß die endlich erhaltenen Produkte noch mit anderen, daneben vorkommenden Substanzen der Nucleinsäuregruppe verunreinigt sind.

Am auffallendsten sind diese Verhältnisse bei den Nucleinsäuren der Pankreasdrüse. Die Angabe Bangs, daß hier eine der echten Nucleinsäure nahestehende, aber von ihr verschiedene Substanz, die Guanylsäure, vorkommen sollte, war, besonders seitdem A. Neumann ${ }^{1}$ ) und P. A. Levene ${ }^{2}$ ) Mitteilungen über eine «Thymonucleinsäure» im Pankreas gemacht hatten, von manchen Forschern angezweifelt worden;

1) Arch. Physiol., 1899, Suppl.-Bd. S. 555.

2) Diese Zeitschrift, Bd. 32, S. 540; Bd. 37, S. 402; Bd. 43, S. 199. 
es herrschte eine große Verwirrung, die erst beseitigt wurde, als H. Steudel ${ }^{1}$ ) darauf aufmerksam gemacht hatte, daß neben der Guanylsäure auch eine zur Gruppe der echten Nucleinsäure gehörige Substanz in der Pankreasdrüse vorkommt.

Eine genauere Charakteristik dieses Körpers als echte Nucleinsäure, so wie sie heute verlangt werden muß, ist aber noch nicht erfolgt. Zwar hat Levene in der nach seiner Methode isolierten Pankreasnucleinsäure sämtliche Spaltprodukte gefunden, wie sie auch aus der echten Nucleinsäure erhalten werden; seine Analysenwerte ${ }^{2}$ ) stimmen jedoch bei den Verhältnissen $\mathrm{N}$ : $\mathrm{P}$ nicht mit den für eine echte Nucleinsäure geforderten Daten überein. So hat er in seinen Präparaten ein Verhältnis $N$ : $P$ gefunden, das zwischen 1,85 und 2,00 schwankt, während bei der echten Nucleinsäure ein Verhältnis $\mathrm{N}: \mathrm{P}=1,70$ verlangt wird.

Zur genaueren Untersuchung der Pankreasnucleinsäure stand mir in unserem Institute ein reiches Ausgangsmaterial zur Verfügung. Es waren nämlich schon seit Jahren die nach der Verarbeitung der Pankreasdrüsen auf das Nucleoproteid nach Hammarsten verbleibenden Rückstande von annähernd 1000 Drüsen jedesmal nach dem Neumannschen Verfahren verarbeitet worden. Die gesammelten Alkoholniederschläge mußten also - neben Resten der Guanylsäure - auch die Nucleinsäure enthalten. Über das Verfahren der Reindarstellung derselben werde $i c h$, da voraussichtlich bei den mannigfach sich ergebenden Schwierigkeiten eine Änderung in dem einen oder anderen Punkte als notwendig sich erweisen wird, später berichten. Weil aber der von mir isolierte Körper sich in mehreren wesentlichen Punkten von der aus der Thymusdrüse gewonnenen echten Nucleinsäure unterscheidet, so soll auf diese Unterschiede schon jetzt eingegangen werden.

Zunächst ist das Verhalten der Pankreasnucleinsäure zu Krystallviolett ein ganz anderes wie das der echten Nucleinsäure. Ich hábe gezeigt, $\left.{ }^{3}\right)$ daß das nucleinsaure Krystallviolett

1) Diese Zeitschrift, Bd. 53, S. 539.

2) Diese Zeitschrift, Bd. 32, S. 540.

3) Diese Zeitschrift, Bd. 84, S. 320. 
in methylalkoholischer Lösung sehr leicht etwa die Hälfte seines Farbbasengehaltes unter Bildung eines sauren Salzes abgibt, sodaß man mit Hilfe der nachfolgenden Amylalkoholfällung einen Körper von ganz anderen Eigenschaften und ganz anderer Zusammensetzung bekommt. Vor der Amylalkoholfällung lag ganz zweifellos ein neutrales Salz der vierbasischen echten Nucleinsäure vor. Dies geht sowohl aus den Analysenzahlen als auch aus der Menge der in dem Salze enthaltenen Farbbase hervor, die sich mit Alkali, weil in Wasser unlöslich, leicht in fester Form abscheiden und wägen läßt. Nach der Lösung des Farbnucleinates im Methylalkohol und Fällung mit Amylalkohol war jedoch, wie sich aus zwei ge- trennt dargestellten Präparaten ergab, das vorher auf das neutrale Salz stimmende Verhältnis N:P von 3,05 auf durchschnittlich 2,4 herabgesunken; außerdem enthielt das derart umgefällte Nucleinat nicht mehr wie vorher ca. 50\% Farbbase, sondern nur noch $20-30 \%$. Dem Austritte der Hälfte des Farbbasengehaltes entsprechend änderten sich auch die äußeren Eigenschaften des umgefällten Körpers. Das ursprüngliche neutrale vierbasische Farbnucleinat war grobkörnig, sehr hart und zeigte die 0 berflächenfarbe des Krystallvioletts (grünlich); das durch Umfällung aus methylalkoholischer Lösung erhaltene saure Salz hingegen war äußerst leicht und locker, sowie mühlos zu feinstem Pulver zerdrückbar. Seine Farbe war im Gegensatze zu dem neutralen Salze nicht grünlich, sondern prächtig blau-violett. Dieser Körper löste sich dann nicht mehr in absolutem Methylalkohol.

Wurde nun in ganz der gleichen Weise das analoge Farbsalz der Pankreasnucleinsäure gebildet, in Methylalkohol gelöst und mit Amylalkohol wieder gefällt, so wurde eine Substanz erhalten, die sich weder hinsichtlich der Analysenwerte noch in ihrem äußeren Verhalten von dem neutralen Salze vor der Fällung unterschied. Der umgefällte Körper war ebenso grün gefärbt wie vorher, er löste sich auch nach der Amylalkoholfällung wieder mit Leichtigkeit in Methylakohol $\mathrm{zu}$ einer tiefblauen Farblösung restlos auf; und dieses Auflösen und Wiederausfällen konnte - im Gegensatze zu dem Farb- 
salze der echten Nucleinsäure - beliebig oft wiederholt werden. Das ermittelte Verhältnis $N: P$ zeigte keinen Abfall wie bei dem Farbsalze der echten Nucleinsäure, und endlich enthielt der umgefällte Körper ebensoviel Farbbase - nämlich etwa $50 \%$ - wie vorher.

Pankreasnucleinat. Präparat I.

A. Vor der Auflösung (nicht analysiert).

B. Nach der Amylalkoholfällung.

$$
\begin{aligned}
& \text { 1. } \mathrm{N}=12,76 \% \\
& \text { 2. } \mathrm{P}=4,26 \% \quad \text { Mittel }=4,24 \% \\
& \text { 3. } \mathrm{P}=4,22 \% \\
& \mathrm{~N}: \mathbf{P}=2,90 \text {. }
\end{aligned}
$$

Pankreasnucleinat. Präparat II.

A. Vor der Auflösung.

$$
\begin{aligned}
& \text { 4. } \mathrm{N}=10,93 \% \\
& \text { 5. } \mathrm{P}=3,46 \% \text { Mittel }=3,45 \% \\
& \text { 6. } P=3,43 \% \\
& \mathbf{N}: \mathbf{P}=\mathbf{3 , 1 7} \text {. }
\end{aligned}
$$

B. Nach der Amylalkoholfällung.

$$
\begin{aligned}
& \text { 7. } \mathrm{N}=12,10 \% \\
& \text { 8. } \mathrm{P}=4,00 \% \text {. Mittel }=3,93 \% \\
& \text { 9. } \mathrm{P}=3,87 \% \\
& \mathbf{N}: \mathbf{P}=\mathbf{3 , 0 8} \text {. }
\end{aligned}
$$

Diese Präparate sind lufttrocken analysiert.

Einen weiteren Unterschied ergab das Resultat der Schwefelsäurespaltung. Es standen mir zu diesem Zwecke etwa $27 \mathrm{~g}$ Pankreasnucleinsäure zur Verfügung. Von den Spaltungsprodukten, die auch in der echten Nucleinsäure aus der Thymus vorkommen, konnten nur Adenin, Cytosin, Thymin, Lärulinsäure und Phosphorsäure in befriedigender Ausbeute (durchschnittlich 1-2 g) erhalten und identifiziert werden. Die Ausbeute an Guanin betrug indessen nur $0,2 \mathrm{~g}$, während theoretisch etwa $2,5 \mathrm{~g}$ hätten erhalten werden müssen. Diese auffallende Beobachtung legte den Gedanken nahe, daß in der 
Pankreasnucleinsäure überhaupt kein Guanin vorkomme, und daß die geringen gefundenen Mengen lediglich von der noch beigemengten Guanylsäure herrührten. Es wurde darauf von mir ein Verfahren ausgearbeitet, welches eine sichere Trennung von Nucleinsäure und Guanylsäure gestattet. Die an derartigen Präparaten vorgenommenen Untersuchungen auf Guanin sind bisher stets negativ verlaufen. Da die von mir geübte Darstellungsweise der Pankreasnucleinsäure eine sehr schonende ist - es wurde $z$. B. stets bei schwach alkalischer Reaktion im Vakuum eingedampft -, so ist eine nachträgliche Abspaltung von Guanin, wie sie ja in mineralsaurer Lösung sehr leicht sich vollzieht, völlig ausgeschlossen. Ich habe folgende Proben auf Guanin angestellt:

1. Spaltung der Pankreasnucleinsäure mit Salpetersäure in der Kälte nach $\mathrm{H}$. Steudel. $10 \mathrm{~g}$ Nucleinsäure wurden mit $20 \mathrm{ccm}$ Salpetersäure vom Sp. G. 1,2 stehen gelassen, bis eine Abscheidung von unlöslichen Purinnitraten nicht mehr stattfand. Die Nitrate wurden sodann abfiltriert, in wenig verdünnter Natronlauge gelöst, mit Essigsäure angesäuert und darauf mit Ammoniak übersättigt: Es trat auch nach längerem Stehen kein Niederschlag von Guanin auf.

2. $2 \mathrm{~g}$ Pankreasnucleinsäure wurden mit 5\% iger Schwefelsäure durch fünfstündiges Kochen gespalten und die Hydrolysenflüsssigkeit sodann mit Ammoniak übersättigt: Es entstand kein Niederschlag von Guanin, während ein sulcher unter den gleichen Bedingungen aus echter Nucleinsäure mit Leichtigkeit erhalten werden konnte.

3. $5 \mathrm{~g}$ Pankreasnucleinsäure wurden durch fünfstündiges Kochen mit 5\% iger Schwefelsäure gespalten, die Acidität der Hydrolysenflüssigkeit mit Natronlauge auf etwa den vierten Teil zurückgebracht und die Purine durch Digerieren der Flüssigkeit mit festem Silbersulfat in der Wärme niedergeschlagen. Nach dem Erkalten wurden die Silberpurine abfiltriert, mit 10\% iger Salzsäure zerlegt und das Filter samt Rückstand (Chlorsilber) wiederholt mit Salzsäure ausgekocht. Die salzsaure Lösung der Purinchloride wurde dann zur Trockene gebracht, der Rückstand in Natronlauge gelöst, mit Essigsäure 
schwach angesäuert und dann mit Ammoniak bis zu einem Gehalte von $2 \%$ übersättigt. Es entstand beim Übersättigen mit Ammoniak ein minimaler Niederschlag, der in Natronlauge nicht löslich wàr und offenbar aus Huminsubstanzen bestand. Er wog nur $50 \mathrm{mg}$ und hatte einen Stickstoffgehalt von zirka $8,8 \%$, bestand also sicherlich nicht aus Guanin, da Guanin annähernd $45 \% \mathrm{~N}$ enthält. Aus der echten Nucleinsäure konnten in einem Parallelversuch mit dieser Methode 0,3 $\mathrm{g}$ Guanin erhalten werden; berechnet sind $0,5 \mathrm{~g}$.

Nach diesen Untersuchungen halte ich es für möglich, daß die Pankreasnucleinsäure überhaupt kein Guanin enthält. Da mir aber bisher nur verhältnismäßig bescheidene Mengen des kostbaren Materials zur Verfügung gestanden haben, von denen der größte Teil zudem zur Ausarbeitung der Methoden verbraucht wurde, so gebe ich diese Resultate besonders in Anbetracht der $\mathrm{W}$ ichtigkeit der Frage mit Vorbehalt wieder.

Unter der Voraussetzung, daß der ganze zum Guanin gehörige Komplex: Phosphorsäure-Hexose-Guanin in der Pankreasnucleinsäure fehlt, würde sich aus dem noch verbleibenden Reste, entsprechend dem Vorhandensein von noch drei Atomen Phosphor und zehn Atomen $\mathrm{N}$, ein Verhältnis $\mathrm{N}: \mathrm{P}=1,51$ ergeben. Gefunden wurde von mir für $\mathrm{N}: \mathrm{P}$ im Mittel 1,53, wie sich aus nachfolgenden Analysenzahlen ergibt:

(Die Präparate sind wieder lufttrocken analysiert.)

\section{Präparat I.}

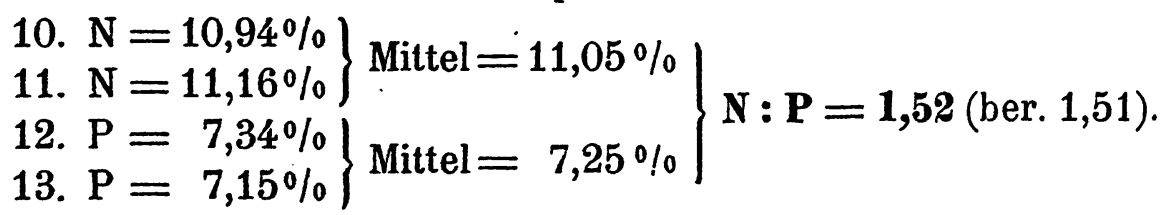

Präparat II.

$\left.\begin{array}{l}\left.\text { 14. } \begin{array}{l}\mathrm{N}=12,46 \% \\ \text { 15. } \mathrm{N}=12,48 \% \\ \text { 16. } \mathrm{P}=7,97 \% \\ \text { 17. } \mathrm{P}=8,00 \% \\ \text { 18. } \mathrm{P}=8,22 \%\end{array}\right\} \text { Mittel }=12,47 \%\end{array}\right\} \mathrm{N}: \mathbf{P}=\mathbf{1 , 5 5}$ (ber. 1,51).

Die Untersuchungen werden von mir fortgesetzt. 
Analytische Belege.

1. $0,2844 \mathrm{~g}$ lieferten $30,8 \mathrm{ccm} \mathrm{N} ; \mathrm{t}=19^{\circ}, \mathrm{b}=.758 \mathrm{~mm}, \mathrm{~N}=12,76 \%$

2. 0,1744 , sättigten $13,4, n / 2$-Lauge; $P=4,26 \%$ (Neumann)

$3.0,1788,>13,6>P=4,22 \%$

4. 0,3865 , lieferten 36,2 , $N ; t=16^{\circ}, b=759 \mathrm{~mm}, \mathrm{~N}=10,93 \%$

5. 0,2021 > sättigten 12,6 > $/ 2$-Lauge; $P=3,46 \%$ (Neumann)

$6.0,1943>>12,0>>P=3,43 \%$,

7. 0,2058 , lieferten $21,8>\mathrm{N} ; \mathrm{t}=18^{\circ}, \mathrm{b}=750 \mathrm{~mm}, \mathrm{~N}=12,10 \%$

8. 0,1554 s sättigten $11,2 \rightarrow n / 2$-Lauge; $P=4,00 \%$ (Neumann)

9. $0,1677>>11,7$ > $>\quad P=3,87 \%$

10. $0,2074,>16,2>n / 10-$ Säure; $N=10,94 \%$ (Kjeldahl)

11. $0,2209,>17,6>\quad N=11,16 \%$,

12. $0,1043>>13,8>\mathrm{n} / 2$-Lauge; $\mathrm{P}=7,34 \%$ (Neumann)

13. $0,1186,>15,3, \quad, P=7,15 \%$,

14. 0,2755 > 24,5 $\gg \mathrm{n} / 10$-Säure; $N=12,46 \%$ (Kjeldahl)

15. 0,2535 > $>22,6>N=12,48 \%$,

16. $0,1065, \quad 15,3, \mathrm{n} / 2$-Lauge; $\mathrm{P}=7,97 \%$ (Neumann)

17. 0,1228 , > 17,7 , $, P=8,00 \%$,

$18.0,1025,>15,2, \quad>\quad P=8,22 \%$, 